\title{
Effect of acupuncture for constipation after ischemic stroke: study protocol for a randomized controlled trial
}

Tao Zhang ${ }^{1}$, Guiling Wang ${ }^{1}$, Bin $\mathrm{Li}^{1}$, Linpeng Wang ${ }^{1}$, Jing Guo', Junxia Hu', Xin Du', Qiuyang Hong², Jingqing Sun ${ }^{1 *+}$ and Cunzhi Liü ${ }^{3 *+}$

\begin{abstract}
Background: Constipation is a common complication after stroke that can severely influence a patient's quality of life and rehabilitation. Treatments for constipation after stroke vary. Acupuncture may improve spontaneous bowel movements, quality of life, and clinical symptoms. The study seeks to assess the preliminary effects of acupuncture on constipation after an ischemic stroke.

Methods/design: This is a prospective randomized controlled pilot trial design in which 120 eligible patients will be randomly allocated to one of three groups. The acupuncture group $(n=40)$ will receive acupuncture and routine care, the medication group $(n=40)$ will receive mosapride citrate and routine care, and the control group $(n=40)$ will receive only routine care for ischemic stroke. Patients will be recruited 2 weeks to 6 months after stroke onset and will receive the intervention continuously over 4 weeks, with a follow-up period of 4 additional weeks. Adverse events will be recorded to assess the safety and tolerability of acupuncture for constipation after an ischemic stroke. The primary outcome will be the change in the weekly mean number of complete spontaneous bowel movements. Secondary outcomes will include any change in the weekly mean number of spontaneous bowel movements, mean stool consistency scores, mean straining scores during defecation, and frequency of laxative use. All outcome measures will be assessed at inception, after the intervention (4 weeks), and at the follow-up (8 weeks).
\end{abstract}

Discussion: This study will provide evidence of the preliminary effects and inform future sample size calculations for studies of acupuncture for constipation following an ischemic stroke. These findings will inform subsequent large-scale randomized controlled trials.

Trial registration: ISRCTN, 22214747. Registered on 17 August 2015.

Keywords: Acupuncture, Constipation, Ischemic stroke, Study protocol

\section{Background}

Globally, stroke is the second leading cause of mortality and disability among adults over 60 years old $[1,2]$. Due to an aging population, dietary changes, pronounced work-related stress, and rapid economic development, there is an increasing prevalence of stroke among younger individuals [3]. Constipation is a common

\footnotetext{
* Correspondence: sjq100037@sina.com; Icz623780@126.com

${ }^{\dagger}$ Jingqing Sun and Cunzhi Liu contributed equally to this work.

'Department of Acupuncture and Moxibustion, Beijing Hospital of Traditional

Chinese Medicine affiliated to Capital Medical University, Beijing, China

${ }^{3}$ Dongfang Hospital affiliated to Beijing University of Traditional Chinese Medicine, Beijing, China

Full list of author information is available at the end of the article
}

complication after stroke [4]. Population-based studies indicate that approximately $30 \%$ to $60 \%$ of stroke patients suffer, or have suffered, from constipation following stroke [5-8]. This wide range can likely be attributed to the adoption of different time points $[5,7,8]$, the diagnostic criteria used to measure constipation $[4,5]$ and patient characteristics [5, 6]. Constipation can severely impair a patient's quality of life and rehabilitation [9]. Consequently, constipation incurs a heavy burden on patients, patients' families, and society as a whole [10].

Treatments for constipation after stroke vary and include lifestyle changes [11-13], diet changes [14-18], use of laxatives, enemas, prokinetic drugs, and surgically

(c) The Author(s). 2018 Open Access This article is distributed under the terms of the Creative Commons Attribution 4.0 International License (http://creativecommons.org/licenses/by/4.0/), which permits unrestricted use, distribution, and reproduction in any medium, provided you give appropriate credit to the original author(s) and the source, provide a link to the Creative Commons license, and indicate if changes were made. The Creative Commons Public Domain Dedication waiver (http://creativecommons.org/publicdomain/zero/1.0/) applies to the data made available in this article, unless otherwise stated. 
implanted neuro-modulation [19]. Unfortunately, many of these treatments are poorly tolerated or rejected, or there may be a lack of clinical evidence supporting their use. Thus, there is a need for a safe, effective, and non-toxic therapy for constipation after a stroke.

Acupuncture has a history of more than 2000 years in China and plays an important role in traditional Chinese medicine [20]. A recent systematic review indicated that acupuncture is safe and tolerable, and may improve weekly spontaneous bowel movements (SBMs), quality of life, and constipation symptoms [21]. Acupuncture may, therefore, be an effective treatment for post-stroke constipation and it has several advantages compared with other therapies [22]. However, using acupuncture to treat constipation has not been validated with high-quality clinical evidence. In addition, clinical research focusing on acupuncture for constipation after stroke is rare [19]. Therefore, we designed this trial to assess the preliminary effects and inform future sample size calculations for studies of acupuncture for constipation following an ischemic stroke. Our hypothesis is that the effectiveness of acupuncture is no less than that of mosapride and better than routine health care.

\section{Methods/design}

\section{Study design}

This is a prospective parallel-arm randomized controlled pilot trial comparing acupuncture with a pharmacological intervention (mosapride citrate) and usual care (control group). We will recruit patients who have sustained an ischemic stroke and present with constipation, according to predefined inclusion and exclusion criteria, between January 2017 and September 2019. Participants will be randomly allocated to one of three groups and will receive continuous treatment for 4 weeks. Repeat assessments will take place after treatment and later, at 8 weeks after inception. All participants will be asked to keep a defecation diary including weekly number of complete spontaneous bowel movements (CSBMs), SBMs, stool consistency, defecation difficulty, and laxative use and dosage, beginning at baseline during the 8-week assessment period. All outcomes will be assessed at baseline, and at 4- and 8-weeks after randomization, according to the defecation diary (Fig. 1).

\section{Randomization and allocation concealment}

Participants will be randomly assigned to one of three groups (the acupuncture group, the medication group,

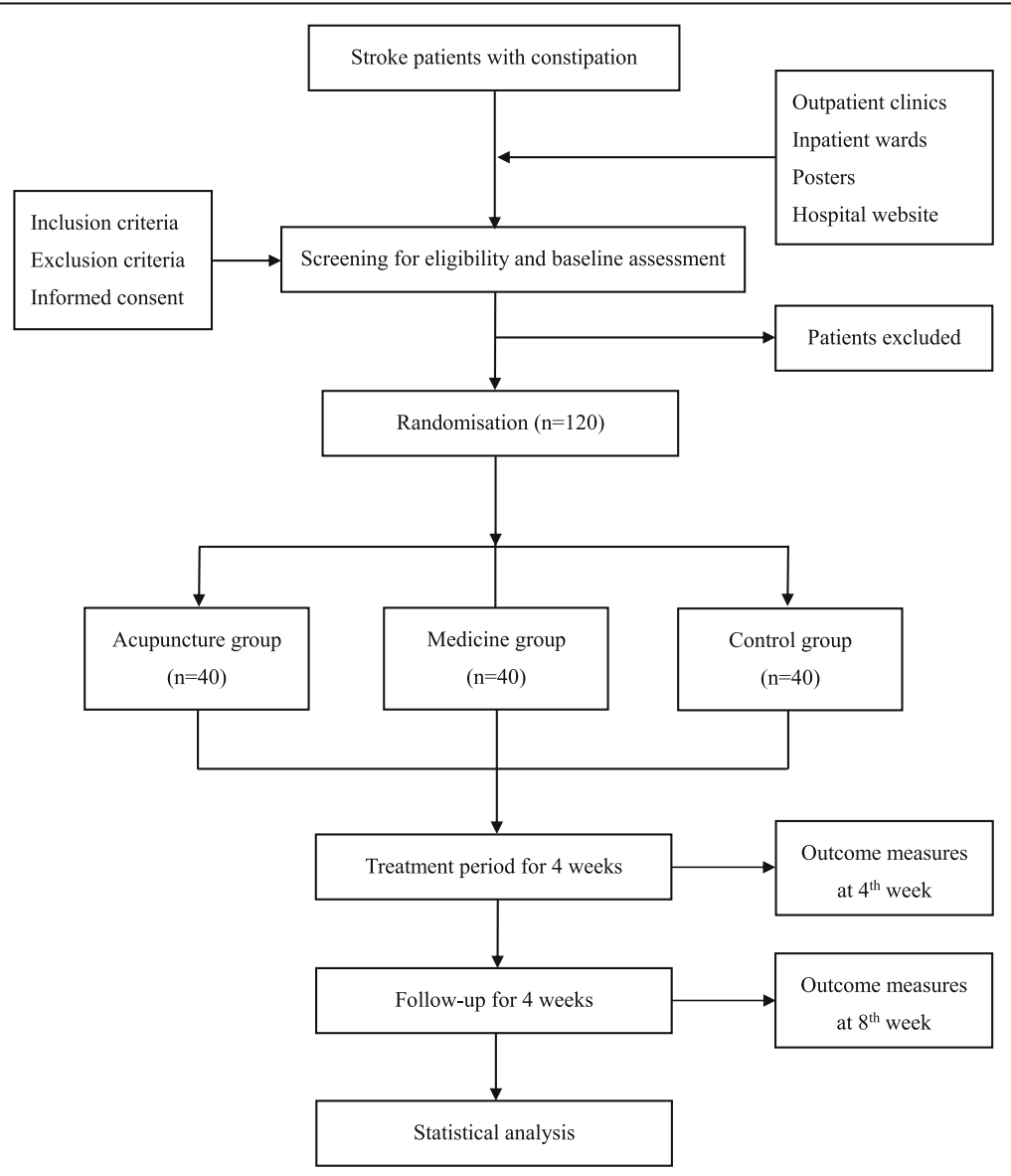

Fig. 1 Trial flow chart 
or the control group) using a 1:1:1 ratio. The generation and allocation of a random sequence for group assignment will be conducted by the Research Centre of Clinical Epidemiology, which is affiliated to Peking University Third Hospital in China. Individuals involved in participant randomization will be blinded and will not participate in the recruitment and inclusion of participants. The block randomization method (block size of 6) will be adopted to generate the random sequence, using the Statistical Analysis System (V.9.1.3; SAS Institute Inc., Cary, North Carolina, USA). Opaque computergenerated sealed envelopes will be produced for the randomization and allocation. The envelopes will be numbered consecutively with a serial number on the outside and will contain the allocation information. An envelope will be opened when a participant enters the study after the baseline measurements have been taken. Participants will be allocated to one of the three groups and receive an intervention according to their group allocation. The random allocation sequence and the opaque sealed envelopes will be kept separately by two specific researchers.

\section{Blinding}

Data managers (including the telephone interviewer during the follow-up period) and statisticians will be blinded to participant group assignment. Therapists, data managers, and statisticians will not be allowed to communicate with others about the participants' allocation.

\section{Participants}

\section{Study population}

Patients with ischemic stroke who present with constipation will be recruited from the acupuncture and moxibustion departments of the Beijing Hospital of Traditional Chinese Medicine Affiliated to Capital Medical University in Beijing, China.

\section{Sample size}

Since there are no previous three-armed randomized controlled trials that investigate this topic, the present study will provide effect size data for sample size calculations of subsequent large-scale randomized controlled trials. For practical reasons, a sample size of 40 per group and a total number of 120 participants will be recruited during the entire study according to outpatient and inpatient censuses of the hospital.

\section{Recruitment and baseline assessment}

Researchers will recruit participants from outpatient clinics and inpatient wards of the acupuncture and moxibustion departments. Recruitment posters will be posted in the hospital. The posters will contain a brief, Institutional Review Board-approved introduction to the study, the participants, the interventions, and contact information for the researchers. The recruitment information will also be uploaded to the hospital website (http:// www.bjzhongyi.com).

Consent will be obtained by one of the investigators, with a witness present. Following written informed consent, eligible patients will be asked to complete a general information form including name, gender, age, medical history, etc. and complete a 1-week defecation diary as the baseline assessment. A neurologist will determine the Glasgow Coma Scale score and the National Institute of Health Stroke Scale score for each participant and these scores will be used to decide whether to include the patient in the study according to the inclusion and exclusion criteria. Personal information about potential and enrolled participants will be collected and maintained confidentially.

\section{Inclusion criteria}

Inclusion criteria are a diagnosis of ischemic stroke according to the diagnostic criteria specified by the World Health Organization [23], constipation symptoms according to the Rome IV Functional Constipation Criteria [24], 2 weeks to 6 months after stroke onset, aged between 30 and 75 years old, Glasgow Coma Scale score $\geq 7$ and National Institute of Health Stroke Scale $\leq 21$, no previous medical history of constipation before stroke onset and symptoms of constipation present for at least 2 consecutive weeks after stroke, no use of gastrointestinal drugs 1 week before randomization (except for emergency drugs), no acupuncture treatment for constipation in the previous 2 weeks, and signed informed consent form.

\section{Exclusion criteria}

Patients with any of the following conditions will be excluded: irritable bowel syndrome or constipation secondary to organic diseases (e.g. endocrine, metabolic, or postoperative diseases); abdominal aneurysms, hepatosplenomegaly, or serious cardiovascular, liver, kidney, or psychiatric diseases; and blood coagulation disorders. Also excluded are pregnant or lactating women and patients requiring administration of anticoagulant agents, such as warfarin or heparin. Participants treated with antiplatelet treatments, such as aspirin or clopidogrel, for the secondary prevention of cerebral infarction are eligible.

\section{Intervention}

The three groups will receive routine health care and secondary prevention as for all other inpatients with ischemic stroke. These treatments include antiplatelet therapies, antihypertensive therapies, glucose control, dyslipidemia control, and dehydration treatments, if necessary. For participants with no SBMs over 4 or more days, use of laxatives (regarded as emergency drugs, 
such as bisacodyl, glycerin enema etc.) will be permitted as an emergency measure to promote defecation. All information regarding the use of emergency drugs (including date of administration, and laxative types and dosage) will be recorded. Before the initiation of the study, all researchers will receive standardized training regarding the content of the trial, treatment strategies, assessments, and quality control. Interventions will be performed in accordance with the STRICTA [25] and good clinical practice guidelines.

\section{Acupuncture group}

All the study acupuncturists will be registered practitioners of traditional Chinese medicine with at least 5 years' clinical experience, as well as a medical education background. The acupuncture treatments and manipulations will be standardized among acupuncture practitioners through live and recorded training. Outpatients will receive treatment in clinics and inpatients will be treated in wards. If inpatients are discharged, they will receive acupuncture in clinics by the same acupuncture practitioner. Sterile needles (size $0.30 \times 40 \mathrm{~mm}$ and $0.35 \times 75 \mathrm{~mm}$; Guizhou Ande Medication Appliance, Ltd., Guiyang City, Guizhou Province, China) will be used.

For the acupuncture, we will use Wang's classical 10 acupoints formula. These acupoints have been selected and combined for gastrointestinal disease after a stroke based on the clinical experience of an accomplished Chinese acupuncturist, Leting Wang, in the 1960s [26, 27]. The 10 acupoints include CV13 (shangwan), CV12 (zhongwan), CV10 (xiawan), bilateral ST25 (tianshu), CV6 (qihai), bilateral PC6 (neiguan), and bilateral ST36 (zusanli). All of the acupoints will be located according to the World Health Organization Standardized Acupuncture Points Location [28]. After sterilizing the skin, needles will be slowly and vertically inserted into ST25 (tianshu) bilaterally, without any manipulation. Needle insertion will stop upon piercing of the muscle layer of the abdominal wall (nearly 20 to $60 \mathrm{~mm}$ subcutaneously). Known side effects of needle insertion include mild soreness. Other needles will be inserted to a depth of $10-25 \mathrm{~mm}$ to predetermined acupoints and manually manipulated by rotation to produce the characteristic sensation of deqi. The deqi sensation is also called needle sensation, and refers to perceived subcutaneous tension around the needle, which is felt by the acupuncture practitioner, and soreness, numbness, distension, and heaviness around the acupuncture point that is felt by the participant. Each session will last for approximately 30 min. All needles will be removed with sterile cotton balls to avoid bleeding.

Each participant will be treated once a day and will receive 16 treatment sessions in total over 4 consecutive weeks. Treatment will be conducted 5 times per week in the first 2 weeks and 3 times per week in the last 2 weeks.

\section{Medication group}

Participants in the medication group will receive $5 \mathrm{mg}$ of mosapride citrate, 3 times per day for 4 consecutive weeks. Unused tablets will be collected by the researchers at the end of the treatment period.

\section{Control group}

Participants in the control group will receive routine health care and secondary prevention treatments for ischemic stroke. For participants with no SBMs over 4 or more consecutive days, cathartic treatment will be given. Details related to the usage of cathartic drugs will be recorded during the trial.

\section{Follow-up}

After the 4-week treatment, all participants will enter an additional 4-week follow-up period. During this time, they will receive routine health care and secondary prevention as provided to all other patients with ischemic stroke. Laxatives will be allowed for use as emergency drugs during the follow-up period. However, acupuncture treatment and mosapride citrate are not permitted during follow-up.

\section{Outcome measures \\ Primary outcome measure}

The primary outcome measure will be the change in the weekly mean number of CSBMs over the 4 weeks of the intervention and the 4 weeks of the follow-up. A CSBM is defined as a SBM unrelated to the administration of laxatives or finger manipulation, accompanied by a self-reported feeling of complete evacuation. Bowel movements achieved with the help of glycerin, enemas, or finger manipulation are not considered spontaneous. If bowel movements occur within $24 \mathrm{~h}$ after the intake of laxatives, they will not be considered spontaneous. However, if bowel movements occur more than $24 \mathrm{~h}$ after administration of laxatives, they will be considered spontaneous. The total number of CSBMs during the treatment period and follow-up period will be averaged to obtain mean weekly CSBMs.

\section{Secondary outcome measures}

1. The change in the number of mean SBMs that are unrelated to the administration of laxatives or finger manipulation, with or without a self-reported feeling of complete evacuation. This measure will be calculated in the same way as the CSBMs.

2. The change in mean stool consistency score, as per the Bristol Stool Scale (Table 1). The score will be 
Table 1 Bristol Stool Scale

\begin{tabular}{ll}
\hline Type & Description \\
\hline 1 & Separate hard lumps, like nuts (hard to pass) \\
2 & Sausage-shaped, but lumpy \\
3 & Like a sausage but with cracks on its surface \\
4 & Like a sausage or snake, smooth and soft \\
5 & Soft blobs with clear-cut edges (passed easily) \\
6 & Fluffy pieces with ragged edges, a mushy stool \\
7 & Watery, no solid pieces; entirely liquid \\
\hline
\end{tabular}

recorded according to the stool type (e.g. type $1=1$ point, type $2=2$ points, etc.) to obtain mean stool consistency scores. The Bristol Stool Scale is a widely used form authored by Dr. K Heaton, reader in medicine, University of Bristol.

It can be downloaded from the website https:// www.bladderandbowel.org/bowel/bowel-resources/ bristol-stool-form-scale/ representations of the different types of stool will be included in the patients' diaries and serve as visual comparators. A patient will record the type of stool in their diary according to the picture.

3. The change in mean straining scores during defecation. The severity of straining will be graded using a 5 -point ordinal scale: $0=$ absent, $1=$ mild, $2=$ moderate, $3=$ severe, and $4=$ very severe.

4. The change in weekly frequency of laxative use. This includes the total number of times that laxatives are used during the 4-week treatment period and the 4-week follow-up period. Instances of laxative use will be summed and divided by 4 to obtain the weekly frequency during the two periods.

\section{Adverse events}

Adverse event data will document the occurrence, duration, and severity of adverse reactions (symptoms and signs), and how the event was resolved (or not) during the treatment. For acupuncture, common and expected adverse events include local hematomas; needle breakage; needle retention after treatment; fainting; unbearable prickling, severe pain, or discomfort persisting for more than $1 \mathrm{~h}$ after acupuncture; local infections; abscesses; and deterioration of blood laboratory parameters. All participants will receive routine blood, urine, and stool tests; and electrocardiogram, liver function (alanine transaminase and aspartate transaminase), and kidney function tests (serum creatinine and blood urea nitrogen). These tests will be performed twice after randomization and at the end of the 4-week treatment period. The researchers will report all adverse events to the Research Ethics Committee of Beijing Hospital of Traditional Chinese Medicine Affiliated to Capital
Medical University and treat the participants with relevant conventional therapy or hospitalization if necessary (the participant's allocated intervention will be revealed). Researchers will evaluate the relationship between adverse events and the interventions. The number of adverse events will be recorded and divided by the sample size of each group to calculate the incidence.

The enrolment schedule, treatment, and outcome measures are presented in Fig. 2.

\section{Statistical analysis}

The analysis of the primary outcome will be based on both a full analysis (intention-to-treat analysis) and per-protocol sets (per-protocol analysis). The analyses of secondary outcomes will be based on the per-protocol sets. The full analysis set refer to the participants who are randomized and receive at least one treatment session. Any missing data will be replaced by the last measured value. The per-protocol sets will be defined as the participants who receive all treatment sessions and completed the study according to the protocol.

The statistical analysis will be performed by a statistician who is blinded to participant group allocation and will not be involved in other aspects of the study. The SPSS statistical package (version 18.0, International Business Machines Corporation, China) will be used to analyze the data. All data will be kept in duplicate by specific researchers. The results of the quantitative data analyses will be presented as means \pm standard deviation. The Kolmogorov-Smirnov test will be applied as the normality test for quantitative variables. The Wilcoxon test or analysis of variance (ANOVA) will be used to compare the difference between groups according to the results of the Kolmogorov-Smirnov test. The results for categorical data will be compared with the chi-squared test and presented as a percentage. The statistical significance level will be set at 0.05 (two-sided) with a $95 \%$ confidence interval.

\section{Ethics and dissemination}

The study protocol follows the principles of the Declaration of Helsinki and was approved by the Research Ethics Committee of Beijing Hospital of Traditional Chinese Medicine Affiliated to Capital Medical University on 9 July 2015 (reference 2015BL-041-01). The study was registered with ISRCTN as a current controlled trial (22214747). The results will be disseminated through peer-reviewed publications, a master's thesis, or conference presentations. The data will be anonymized prior to publication to prevent identification of individual participants.

\section{Monitoring}

The study will be regularly monitored by the Data Management and Monitoring Committee of the Good 


\begin{tabular}{|c|c|c|c|c|c|c|c|}
\hline \multirow[b]{3}{*}{ TIMEPOINT } & \multicolumn{7}{|c|}{ STUDY PERIOD } \\
\hline & \multirow{2}{*}{$\begin{array}{c}\begin{array}{c}\text { Enrol- } \\
\text { ment }\end{array} \\
-1 \text { week }\end{array}$} & \multirow{2}{*}{$\frac{\text { Allocation }}{0}$} & \multicolumn{4}{|c|}{ Treatment period } & \multirow{2}{*}{$\begin{array}{c}\begin{array}{c}\text { Follow-up } \\
\text { period }\end{array} \\
8 \text { week }\end{array}$} \\
\hline & & & $\begin{array}{c}1 \\
\text { week }\end{array}$ & $\begin{array}{c}2 \\
\text { week }\end{array}$ & $\begin{array}{c}3 \\
\text { week }\end{array}$ & $\begin{array}{c}4 \\
\text { week }\end{array}$ & \\
\hline \multicolumn{8}{|l|}{ ENROLMENT } \\
\hline \multirow{4}{*}{$\begin{array}{l}\text { Eligibility screen } \\
\text { Informed consent } \\
\text { Baseline } \\
\text { assessment } \\
\text { Allocation }\end{array}$} & $\mathrm{X}$ & & & & & & \\
\hline & $\mathrm{X}$ & & & & & & \\
\hline & $\mathrm{X}$ & & & & & & \\
\hline & & $\mathrm{X}$ & & & & & \\
\hline \multirow{4}{*}{$\begin{array}{l}\text { INTERVEN- } \\
\text { TIONS } \\
\text { Acupuncture } \\
\text { group } \\
\text { Medicine group } \\
\text { Control group }\end{array}$} & & & & & & & \\
\hline & & & $\mathrm{X}$ & $\mathrm{X}$ & $\mathrm{X}$ & $\mathrm{X}$ & \\
\hline & & & $\mathrm{X}$ & $\mathrm{X}$ & $\mathrm{X}$ & $\mathrm{X}$ & \\
\hline & & & $\mathrm{X}$ & $\mathrm{X}$ & $\mathrm{X}$ & $\mathrm{X}$ & \\
\hline \multicolumn{8}{|l|}{ ASSESSMENTS } \\
\hline CSBMs & $\mathrm{X}$ & & & & & $\mathrm{X}$ & $\mathrm{X}$ \\
\hline SBMs & $\mathrm{X}$ & & & & & $\mathrm{X}$ & $\mathrm{X}$ \\
\hline Stool consistency & $\mathrm{X}$ & & & & & $\mathrm{X}$ & $\mathrm{X}$ \\
\hline Straining score & $\mathrm{X}$ & & & & & $\mathrm{X}$ & $\mathrm{X}$ \\
\hline Laxatives use & $\mathrm{X}$ & & & & & $\mathrm{X}$ & $\mathrm{X}$ \\
\hline Adverse events & & & & & & $\mathrm{X}$ & \\
\hline
\end{tabular}

Fig. 2 Enrolment schedule, treatment, and outcome measures. CSBM complete spontaneous bowel movement, SBM spontaneous bowel movement

Clinical Practice Department of Beijing Hospital of Traditional Chinese Medicine Affiliated to Capital Medical University. The Data Management and Monitoring Committee operates independently from trial researchers and has no competing interests. The Data Management and Monitoring Committee will monitor the overall quality and completeness of the data, examine original case report forms, interview researchers, verify the record of adverse events, and confirm that the study complies with the principles of this protocol.

\section{Discussion}

Constipation is a common health problem after an ischemic stroke. Relevant research has indicated that constipation after an ischemic stroke can impair the removal of metabolic waste [29], aggravate the development of atherosclerosis, increase the risk of cerebral vasospasm and neural damage, lead to the detachment of deep vein thrombosis and cause thromboembolism, and induce the recurrence of cardiovascular and cerebrovascular diseases [7, 8]. Constipation may severely impair a patient's rehabilitation and their quality of life. The results of this study will provide preliminary data on the safety, tolerability, and preliminary effectiveness of acupuncture for constipation symptoms after stroke. It will provide evidence for sample size calculations of subsequent large-scale multi-center randomized controlled trials by assessing effect sizes, variability, and the completeness of the data. The study will be a preliminary investigation into the potential enhancement of a currently used intervention and may contribute to changes in future clinical practice.

Acupuncture is popular in China due to its long history, simple administration, therapeutic effects, low cost, and relative safety. It is also increasingly practiced and requested by patients in Western countries as a complementary and alternative medicine [30]. The World Health Organization has drawn up a provisional list of diseases that could potentially be treated with acupuncture, including constipation, stroke, and its sequelae [31, 32]. To treat constipation, we will use Wang's classical 10 acupoints. These 10 points are combined to treat gastrointestinal disease after a stroke and are based on acupuncture theory and decades of clinical experience $[33,34]$. These points were selected because: (1) all 10 acupuncture points have been used for thousands of years in traditional Chinese medicine to treat constipation and other gastrointestinal diseases and (2) from the 
perspective of evidence-based medicine, clinical randomized controlled trials and systematic reviews also show that these acupuncture points, individually or in combination, may be an effective treatment for constipation [21, 35-38].

The objective of this study is to determine the preliminary effects of acupuncture on constipation after an ischemic stroke. The selection of a control group was a major issue in planning the design of the trial. Reported clinical trials of acupuncture for constipation after a stroke are scarce in both English and Chinese research databases. Consequently, we decided to pursue a pragmatic study design. Due to the scarcity of evidence supporting the use of acupuncture for constipation after a stroke, we did not include a sham acupuncture control group. However, if acupuncture proves to be effective in this study, we will then compare acupuncture with a sham treatment as the next step in clarifying the specific effects of acupuncture. Mosapride, a kind of serotonin 5-hydroxytryptamine $4\left(5-\mathrm{HT}_{4}\right)$ receptor agonist, was designated as the positive drug control in the trial. Clinical evidence and guidelines indicate that $5-\mathrm{HT}_{4}$ receptor agonists, such as prucalopride and mosapride, improve the mobility of the gastrointestinal tract and are effective in the management of constipation [39-44]. Since prucalopride is not widely applied in clinical practice in Beijing, we chose mosapride citrate as the drug of choice for the medication group [45, 46].

The study is a prospective randomized controlled pilot trial. Randomization and allocation concealment are applied to avoid selection bias. Blinding is used to minimize performing bias and detection bias. However, methodological limitations still exist in the study. Due to the nature of acupuncture manipulation, the therapists and the participants cannot be blinded in this study. Therapists have to carry out the acupuncture manipulations and control the participant's sensation of needling. In acupuncture trials, genuine double blinding is difficult to achieve, especially in countries with populations aware of acupuncture (Additional file 1).

\section{Additional file}

Additional File 1: SPIRIT 2013 Checklist: Recommended items to address in a clinical trial protocol and related documents. (DOC $122 \mathrm{~kb}$ )

\section{Abbreviations}

5- $\mathrm{HT}_{4}$ : 5-hydroxytryptamine 4; ANOVA: Analysis of variance; CSBM: Complete spontaneous bowel movement; SBM: Spontaneous bowel movement

\section{Acknowledgements}

We would like to acknowledge Dr. Ines Eisner-Janowicz, who is a special researcher at Beijing Hospital of Traditional Chinese Medicine Affiliated with Capital Medical University, who helped us revise this English language manuscript. We would also like to acknowledge the funding support programs for agreeing to sponsor the study, and the reviewers for their helpful comments on the manuscript.
Trial status

This is version 2.0 of the protocol, dated 5 May 2018. Recruitment began on 2 January 2017. Recruitment is anticipated to end on 30 September 2019.

\section{Funding}

The trial is sponsored and funded by the following organizations and funds: Special Funding Support for Capital Clinical Medical Research of Beijing Municipal Science and Technology Commission, China (Z151100004015109); Beijing Key Laboratory of Acupuncture Neuromodulation, Beijing, China (BZ0437); Capital Core Specialty and Beijing National Key Specialty Radiation Project of Traditional Chinese Medicine (1+X+N Project); National Basic Research Program (973 Program) of China (2014CB543203); Funding Support for Fostering Talents of Beijing Municipal Government, China (2017000021469G298); and Funding Support for Fostering Talents of Beijing Hospital of Traditional Chinese Medicine Affiliated to Capital Medical University, China (XX-201702).

\section{Authors' contributions}

TZ and JS conceived the study and prepared the initial protocol. TZ and GW drafted the manuscript. LW and BL made amendments and participated in the design of the protocol. JG, JH, XD, and $\mathrm{QH}$ participated in revising the manuscript. $\mathrm{CL}$ took charge of the statistical analysis and revised the manuscript as the methodologist. All authors read and approved the final manuscript.

Ethics approval and consent to participate

The Research Ethics Committee of Beijing Hospital of Traditional Chinese Medicine Affiliated to Capital Medical University has approved the study protocol (reference 2015BL-041-01). Before randomization, all participants will be requested to provide written informed consent

Consent for publication

Not applicable.

\section{Competing interests}

The authors declare that they have no competing interests.

\section{Publisher's Note}

Springer Nature remains neutral with regard to jurisdictional claims in published maps and institutional affiliations.

\section{Author details}

${ }^{1}$ Department of Acupuncture and Moxibustion, Beijing Hospital of Traditional Chinese Medicine affiliated to Capital Medical University, Beijing, China.

${ }^{2}$ Department of Acupuncture and Moxibustion, Shunyi Hospital affiliated to Beijing Hospital of Traditional Chinese Medicine, Beijing, China. ${ }^{3}$ Dongfang Hospital affiliated to Beijing University of Traditional Chinese Medicine, Beijing, China.

Received: 14 January 2018 Accepted: 19 June 2018

Published online: 22 August 2018

\section{References}

1. Sousa RM, Ferri CP, Acosta D, Albanese E, Guerra M, Huang Y, Jacob KS, Jotheeswaran AT, Rodriguez JJ, Pichardo GR, Rodriguez MC, Salas A, Sosa AL, Williams J, Zuniga T, Prince M. Contribution of chronic diseases to disability in elderly people in countries with low and middle incomes: a 10/66 dementia research group population-based survey. Lancet. 2009;374:1821-30.

2. Lopez AD, Mathers CD, Ezzati M, Jamison DT, Murray CJ. Global and regional burden of disease and risk factors, 2001: systematic analysis of population health data. Lancet. 2006;367:1747-57.

3. Hankey GJ. Stroke: how large a public health problem, and how can the neurologist help? Arch Neurol. 1999;56:748-54.

4. Winge K, Rasmussen D, Werdelin LM. Constipation in neurological diseases. J Neurol Neurosurg Psychiatry. 2003;74:13-9.

5. Robain G, Chennevelle JM, Petit F, Piera JB. Incidence of constipation after recent vascular hemiplegia: a prospective cohort of 152 patients. Rev Neurol (Paris). 2002;158:589-92.

6. Harari D, Norton C, Lockwood L, Swift C. Treatment of constipation and fecal incontinence in stroke patients: randomised controlled trial. Stroke. 2004;35:2549-55. 
7. Su Y, Zhang X, Zeng J, Pei Z, Cheung RT, Zhou QP, Ling L, Yu J, Tan J, Zhang Z. New-onset constipation at acute stage after first stroke: incidence, risk factors, and impact on the stroke outcome. Stroke. 2009;40:1304-9.

8. Bracci F, Badiali D, Pezzotti P, Scivoletto G, Fuoco U, Di Lucente L, Petrelli A, Corazziari E. Chronic constipation in hemiplegic patients. World J Gastroenterol. 2007;13(29):3967-72.

9. Wald A, Sigurdsson L. Quality of life in children and adults with constipation. Best Pract Res Clin Gastroenterol. 2011;25:19-27.

10. Dennison C, Prasad M, Lloyd A, Bhattacharyya SK, Dhawan R, Coyne K. The health-related quality of life and economic burden of constipation. Pharmacoeconomics. 2005;23:461-76.

11. Harari D, Gurwitz JH, Choodnovskiy I, Avorn J, Minaker KL. Correlates of regular laxative use in frail elderly persons. Am J Med. 1995;99:513-8.

12. Ross DG. Altered bowel elimination patterns among hospitalized elderly and middle-aged persons. Orthop Nurs. 1995;14:25-31.

13. Potter JM, Norton C, Cottenden A, editors. Bowel Care in Older People: Research and Practice. London: Royal College of Physicians; 2002.

14. Harari D. Constipation and faecal incontinence in old age. In: Tallis RC, Fillit $\mathrm{SH}$, Brocklehurst TJ, editors. Brocklehurst's textbook of geriatric medicine and gerontology. 6th ed. London: Churchill Livingstone; 2002.

15. Tramonte SM, Brand MB, Mulrow CD, Amato MG, O'Keefe ME, Ramirez G. The treatment of chronic constipation in adults-a systematic review. J Gen Intern Med. 1997;12:15-24.

16. Wong ML, Wee S, Pin CH, Gan GL, Ye HC. Sociodemographic and lifestyle factors associated with constipation in an elderly Asian community. Am J Gastroenterol. 1999;94:1283-91.

17. Towers AL, Burgio KL, Locher JL, Merkel IS, Safaeian M, Wald A. Constipation in the elderly: influence of dietary, psychological, and physiological factors. J Am Geriatr Soc. 1994;42:701-6.

18. Robson KM, Kiely DK, Lembo T. Development of constipation in nursing home residents. Dis Colon Rectum. 2000;43:940-3.

19. Coggrave M, Norton C, Cody JD. Management of faecal incontinence and constipation in adults with central neurological diseases. Cochrane Database Syst Rev. 2014;13:CD002115.

20. Wu JN. A short history of acupuncture. J Altern Complement Med. 1996;2:19-21.

21. Zhang T, Chon TY, Liu B, et al. Efficacy of acupuncture for chronic constipation: a systematic review. Am J Chin Med. 2013;41:717-42.

22. Yang JP, Liu JY, Gu HY, LV WL, Zhao H, Li GP. Randomized controlled trials of acupuncture and moxibustion for post-stroke constipation: a meta analysis. Zhongguo Zhen Jiu. 2014;34(8):833-6.

23. Special report from the National Institute of Neurological Disorders and Stroke. Classification of cerebrovascular diseases III. Stroke. 1990;21:637-76.

24. Lacy BE, Mearin F, Chang L, Chey WD, Lembo AJ, Simren M, Spiller R. Bowel Disorders. Gastroenterology. 2016;150:1393-407.

25. MacPherson $H$, White A, Cummings M, Jobst KA, Rose K, Niemtzow RC, STRICTA Group. Standards for reporting interventions in controlled trials of acupuncture: the STRICTA recommendations. J Altern Complement Med. 2002:8:85-9.

26. Niu XS, Wang F. Mr. Wang Leting: a master of acupuncture. Zhongguo Zhen Jiu. 2012:32:1041-6.

27. Wang G, Cheng J, Sun J. Clinical experience of Leting Wang in the treatment of stroke. Beijing Journal of Traditional Chinese Medicine. 2012;31:23-5.

28. WHO Regional Office for the Western Pacific. WHO Standard Acupuncture Point Locations in the Western Pacific Region. Manila: World Health Organization; 2008

29. Sayama T, Suzuki S, Fukui M. Expression of inducible nitric oxide synthase in rats following subarachnoid hemorrhage. Neurol Res. 1998;20:79-84.

30. NIH Consensus Conference. Acupuncture. JAMA. 1998;280:1518-24.

31. Bonnerman $\mathrm{RH}$. The $\mathrm{WHO}$ viewpoint on acupuncture. Rev Med Interna Neurol Psihiatr Neurochir Dermatovenerol Neurol Psihiatr Neurochir. 1982;27:103-8.

32. World Health Organization. Acupuncture: review and analysis of reports on controlled clinical trials. Geneva: World Health Organization; 2002.

33. Niu XS, Mr WF. Wang Le-ting: a master of acupuncture. Zhongguo Zhen Jiu. 2012;32:1041-6.

34. Wang G, Cheng J, Sun J. Clinical experience of Le-ting Wang in the treatment of stroke. Beijing Journal of Traditional Chinese Medicine. 2012;31:23-5.

35. Li MK, Lee TF. Suen KP. A review on the complementary effects of auriculotherapy in managing constipation. J Altern Complement Med. 2010;16:435-47.

36. Liu Z, Yan S, Wu J, He L, Li N, Dong G, Fang J, Fu W, Fu L, Sun J, Wang L, Wang S, Yang J, Zhang H, Zhang J, Zhao J, Zhou W, Zhou Z, Ai Y, Zhou K, Liu J, Xu H, Cai Y, Liu B. Acupuncture for chronic severe functional constipation: a randomized trial. Ann Intern Med. 2016;165:761-9.
37. Wu J, Liu B, Li N, Sun J, Wang L, Wang L, Cai Y, Ye Y, Liu J, Wang Y, Liu Z. Effect and safety of deep needling and shallow needling for functional constipation: a multicenter, randomised controlled trial. Medicine (Baltimore). 2014;93:e284

38. Xue QM, Li N, Liu ZS, Wang CW, Lu JQ. Efficacy of electroacupuncture in the treatment of functional constipation: a randomised controlled pilot trial. Chin J Integr Med. 2015;21:459-63.

39. Liu Z, Sakakibara R, Odaka T, et al. Mosapride citrate, a novel 5-HT4 agonist and partial 5-HT3 antagonist, ameliorates constipation in parkinsonian patients. Mov Disord. 2005;20:680-6.

40. Ueno $N$, Inui A, Satoh Y. The effect of mosapride citrate on constipation in patients with diabetes. Diabetes Res Clin Pract. 2010;87:27-32

41. Kanazawa M, Watanabe S, Tana C. Effect of 5-HT4 receptor agonist mosapride citrate on rectosigmoid sensorimotor function in patients with irritable bowel syndrome. Neurogastroenterol Motil. 2011;23:754-e332.

42. Ren HY, Luo HS, Chen JH, Xie XP. Hou XH. A randomised double-blind controlled trial of mosapride for patients with functional constipation. Chin J. Dig. 2005;10:614-6.

43. Tack J, Müller-Lissner S, Stanghellini V, Boeckxstaens G, Kamm MA, Simren M, Galmiche JP, Fried M. Diagnosis and treatment of chronic constipation-a European perspective. Neurogastroenterol Motil. 2011;23:697-710.

44. Camilleri M, Kerstens R, Rykx A, Vandeplassche L. A placebo-controlled trial of prucalopride for severe chronic constipation. N Engl J Med. 2008;358:2344-54.

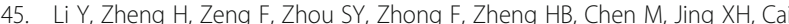
YY, Jia BH, Zhu B, Liu ZS. Use acupuncture to treat functional constipation: study protocol for a randomized controlled trial. Trials. 2012;13:104.

46. Xu X, Zheng C, Zhang M, Wang W, Huang G. A randomized controlled trial of acupuncture to treat functional constipation: design and protocol. BMC Complement Altern Med. 2014;14:423.

\section{Ready to submit your research? Choose BMC and benefit from:}

- fast, convenient online submission

- thorough peer review by experienced researchers in your field

- rapid publication on acceptance

- support for research data, including large and complex data types

- gold Open Access which fosters wider collaboration and increased citations

- maximum visibility for your research: over $100 \mathrm{M}$ website views per year

At BMC, research is always in progress.

Learn more biomedcentral.com/submissions 